\title{
COMPUTATIONAL MHD JETS
}

\author{
Kazunari SHIBATA \\ Department of Earth Sciences, Aichi University of Education, \\ Kariya, Aichi 448, Japan
}

\begin{abstract}
By using two-dimensional magnetohydrodynamic (MHD) code, the following nonsteady MHD jets and outflows are studied in relation to jets ejected from central regions of galaxies: (1) a jet driven by gas pressure gradient, collimated by vertical magnetic fields (as a model of bipolar flows ejected from a hot bubble created by starbursts); (2) a magnetic twist jet which is accelerated and collimated by $\mathbf{J} \times \mathbf{B}$ force in relaxing magnetic twists generated by the interaction of poloidal fields with a rotating disk (as a model of jets from active galactic nuclei); (3) a magnetic-loop-ou tflow resulting from the Parker instability, which may account for the initial acceleration of the magnetic twist jet and nonthermal emissions near nuclear accretion disks.
\end{abstract}

\section{Introduction}

The central regions of galaxies show various types of activity. The most energetic and enigmatic of these are seen in the active galactic nuclei (AGN) with a size smaller than a few parsecs. Well-collimated bipolar radio jets are ejected from these nuclei (e.g., Ferrari and Pacholczyk 1983). Although the detailed structure of the nuclei is not yet known, it is very likely that magnetic fields penetrating accretion disks play an important role on the formation of these radio jets (Lovelace 1976; Blandford and Payne 1982; Shibata and Uchida 1985, 1986, 1987; Uchida and Shibata 1985; Uchida et al. 1985).

Another type of activity called bursting star formation is known in the central region of some galaxies on a scale of a few hundred parsecs. In a typical starburst galaxy M82, many SNRs are observed in the central region of a few 100 pc (e.g., Kronberg et al. 1985), and the explosion or bipolar outflow features at optical, $\mathrm{X}$-ray, and radio wavelengths are considered to be the result of succesive supernova explosions via bursting star formation (Nakai et al. 1987; Sofue 1988). The Galactic center lobe found by Sofue and Handa (1984) may be either a starburst outflow or a low energy prototype of AGN jets (Shibata 1989). Similar elongated flow features are observed also in NGC 3079 (e.g., Duric et al. 1983), and in NGC 253 (Turner 1985). It is again very likely that the collimation of these outflows is due to vertical magnetic fields penetrating the galactic center disk.

In this paper, we study some basic processes in MHD jets and outflows emanating from central regions of galaxies, by using two-dimensional nonsteady MHD numerical simulations.

\section{Gas Pressure Driven Jet Collimated by Vertical Magnetic Fields}

Figure 1 shows a typical example of a gas pressure driven jet which is well collimated by vertical magnetic fields (Umemura et al. 1988). Here, a single adiabatic explosion with energy $E=10^{54} \mathrm{erg}$ is assumed to occur in a pressureless gas disk with density of $10^{-23} \mathrm{~g}$ $\mathrm{cm}^{-3}$ and with vertical magnetic fields of $B=110 \mu \mathrm{G}$. A tenuous halo $\left(\sim 10^{-25} \mathrm{~g} \mathrm{~cm}^{-3}\right)$ is located outside the disk. It is seen that the jet with a velocity of $700 \mathrm{~km} \mathrm{~s}^{-1}$ propagates 


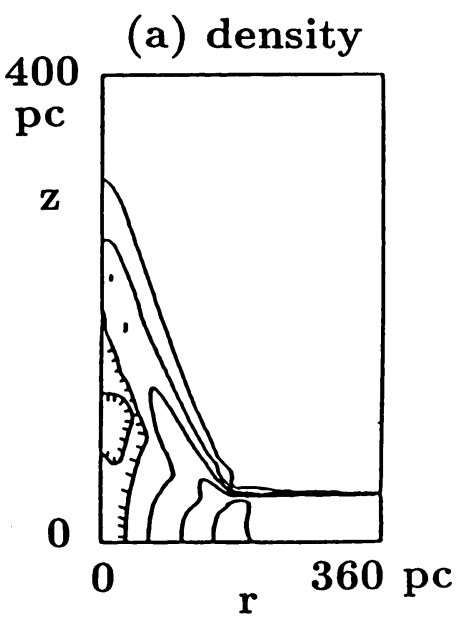

(b) velocity

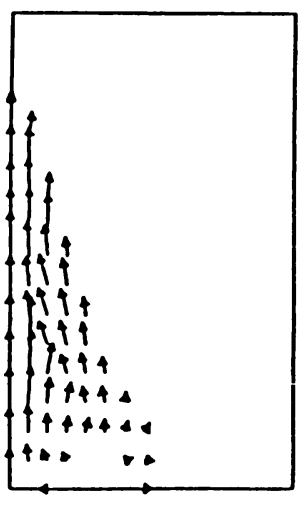

\section{$1300 \mathrm{~km} / \mathrm{s}$}

(c) magnetic field

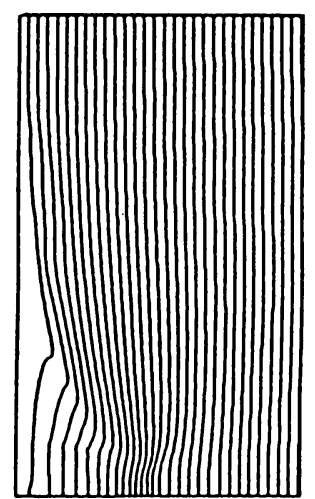

Fig. 1 A gas pressure driven jet collimated by vertical magnetic fields in the case of $B$ $=110 \mu \mathrm{G}$ at $t=4.3 \times 10^{5}$ year (Umemura et al. 1988). (a) density contours, (b) velocity vectors, (c) magnetic field lines. The density contour step width is 0.5 in logarithmic scale, and the unit of velocity vectors are shown below the velocity figure.

(d) $\mathrm{B}$
(a) $\log \rho+V$
(b) $\log \rho+V$
(c) $\mathrm{B}_{p}$
$t=6.50$

$$
t=2.25
$$

$$
\mathrm{t}=6.50
$$

$$
\mathrm{t}=6.50
$$
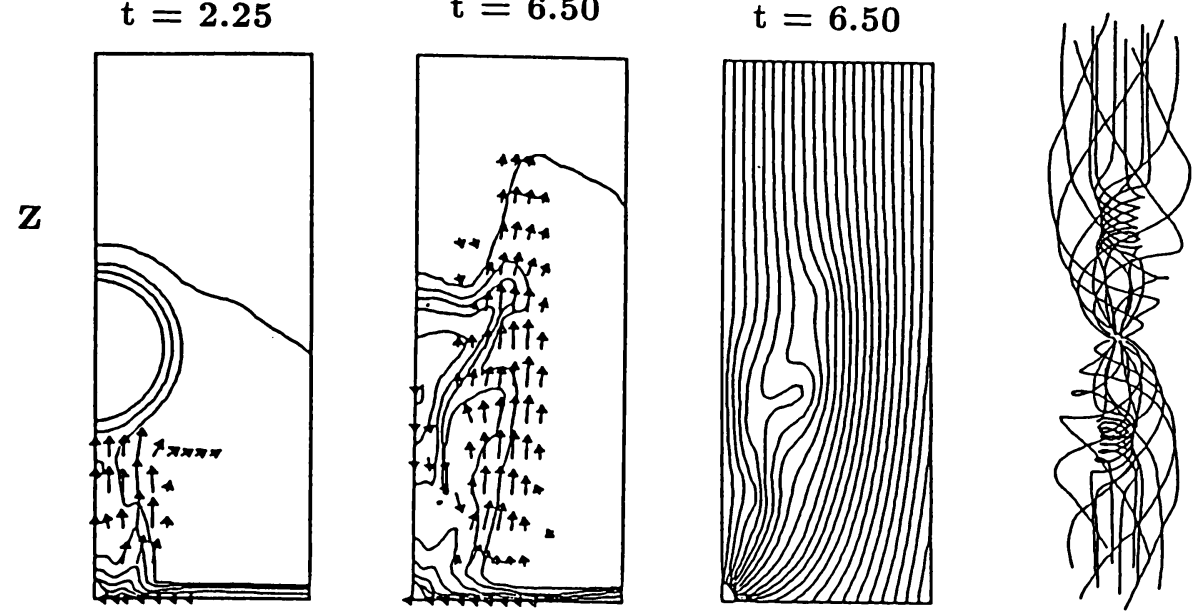

$\mathbf{r}$

Fig. 2 Collision of a magnetic twist jet with a spherical dense blob (cloud) (Shibata and Uchida 1989). The density contour and velocity vectors at (a) $t=2.25$ and (b) 6.50 . (c) The poloidal magnetic field lines. (d) The three dimensional configuration of magnetic field lines. 
almost one-dimensionally along vertical magnetic fields. The gas pressure inside the jet is much smaller than the ambient magnetic pressure, so that the jet is strongly collimated by the magnetic pressure due to poloidal fields. It is also seen that the surface of the jet is a contact surface of the mass ejected from the disk, and a strong reverse fast MHD shock appears inside the jet. (Original forward fast shock propagates farther away from the jet because of a large Alfvén speed in the halo.)

The condition for high collimation of a flow resulting from a single adiabatic explosion in a disk-halo configuration is given by $B \geq 40 \mu \mathrm{G}\left(E / 10^{54} \mathrm{erg}\right)^{1 / 2}\left(R_{0} / 300 \mathrm{pc}\right)^{-3 / 2}$, where $R_{0}$ is the radius of the cross section of the flow.

\section{Magnetic Twist Jet}

Assuming an initial (non-equilibrium) magnetic twist, Shibata and Uchida (1985) studied a magnetic twist jet accelerated by magnetic pressure gradient force in a relaxing magnetic twist, and found that the velocity of the jet is of order of the Alfven speed in the initial magnetic twist; $V_{\text {jet }} \sim 0.1-2.0 V_{A}(t=0)$. The jet is collimated mainly by poloidal fields, although the weak nonlinear sweeping pinch effect is found at the front of the jet.

In a deep central region of AGN, a magnetic twist is created by winding-up of poloidal fields by the differential rotation of an accretion disk. When the twist relaxes along the poloidal fields, a jet is acclerated by the magnetic pressure gradient force in the magnetic twist. By extending the previous study, we have performed numerical simulations of this case (Uchida and Shibata 1985; Shibata and Uchida 1986), and found that the velocity of the jet is comparable to the local Alfven speed in the jet-accelerating region (in the surface layer of the disk) and also of order of the Keplerian velocity $\left(V_{K}\right)$ of the disk just below the jet; $V_{\text {jet }} \sim V_{A}\left(t=t_{1}\right) \sim V_{K}$, where $t_{1}$ is the time when the magnetic twist begins to relax. $\mathrm{Namely}$, the jet starts to be accelerated by the magnetic pressure gradient, when the local Alfvén speed in the surface layer of the disk becomes comparable to the local Keplerian velocity. (The centrifugal force also helps to accelerate the jet.) The dependence of the jet velocity on the initial magnetic field strength is similar to that of the steady centrifugal MHD wind, $V_{\text {jet }} \propto B^{2 / 3}$ (e.g., Pudritz and Norman 1986) or $\propto B$ (e.g., Camenzind 1990). The collimation of the jet near the disk is mainly due to poloidal fields.

However, if initial magnetic fields significantly diverge along $z$-axis, the ratio of toroidal to poloidal magnetic field strengths in the jet substantially increases with $z$, so that the nonlinear pinch effect due to toroidal fields dominates far from the disk (Uchida et al. 1989). In this case, the jet is mainly collimated by toroidal fields. This is very similar to the collimation mechanism in the steady MHD wind (e.g., Lovelace 1990), although our jet is inherently nonsteady, and thus we called it a sweeping-magnetic-twist-jet or a sweeping-pinch-jet (e.g., Uchida and Shibata 1986).

Similar effects of strong toroidal components also appear when a magnetic twist jet collides with a dense blob (Shibata and Uchida 1990). Because the Alfvén speed is low in the blob, magnetic twists are accumulated just in front of the blob. The lower part of the blob is compressed by the pinching force as a result of the nonlinear magnetic twist (Fig.2).

\section{Magnetic-Loop-Outflow as a Result of Parker Instability}

As the gas in the disk contracts toward the inner region of the nucleus, the ratio of toroidal to poloidal fields in the disk gets larger and larger. The disk mass is partly supported by the magnetic pressure force in this toroidal field (Hanawa et al. 1988), and the gas in the surface layer of the disk is eventually accelerated upward by the magnetic pressure to form a magnetic-twist-jet as shown in the previous section. In a realistic three dimensional situation, however, a non-axisymmetric Parker (1966) instability would arise in the nearly toroidal magnetic field. Such a situation is studied using two-dimensional simulation for the local part of a magnetized disk. 
One of the interesting results is the approximate self-similar expansion of a magnetic loop (Shibata et al. 1989, 1990; see Fig. 1 in Shibata and Matsumoto 1990); the rise velocity of the loop increases linearly with height, $V_{\text {loop }} \sim 0.5 \omega z$, where $\omega \sim 0.1 V_{A} / H$ (for $\beta \simeq 1$ ) is the linear growth rate of the Parker instability, $V_{A}$ and $\beta$ are the Alfvén speed and the ratio of gas to magnetic pressure in the initial magnetized layer, and $H$ is the pressure scale height of the disk. It should be noted that the loop density is not smaller than the ambient density in the nonlinear stage, i.e., the loop is not accelerated by the buoyancy force, but is accelerated by the magnetic pressure gradient force.

\section{Conclusion}

We have studied several MHD processes which are considered to be important to understand the acceleration and collimation mechanism of jets and outflows observed in central regions of galaxies. In the largest scale $(\sim 100-400 \mathrm{pc})$, the succesive supernova explosions as a result of the starburst is one of the possible energy source for the large scale, low energy outflows. We stressed the possibility that such flows are collimated by strong vertical magnetic fields (e.g., Morris 1990). In the smaller scale (< a few pc), magnetic fields play more active role on the formation of more energetic jets; $a$ jet is accelerated and collimated by the $\mathbf{J} \times \mathbf{B}$ force in a relaxing magnetic twist which is created by the interaction of poloidal fields with an accretion disk. Angular momentum transfer associated with the production of the magnetic twist jet may resolve the so-called $\alpha$-viscosity problem. In the smallest scale, magnetic fields are tightly wound up by the effect of the differential rotation of the accretion disk. Consequently, the Parker instability occurs to create many expanding magnetic loops, which may explain the initial acceleration of the magnetic twist jet and nonthermal emissions from AGN through the flare-like activity.

\section{References}

Blandford, R. and Payne, D. G. 1982, Mon. Not. Roy. Astron. Soc., 199, 883.

Camenzind, M. 1990, in this volume.

Duric, N., Seaquist, E. R., Crane, P. C., Bignell, R. C., and Davis, L. E. 1983, Ap. J. Lett., 273, L11.

Ferrari, A., and Pacholczyk, A. G. (ed.), Astrophysical Jets, Reidel.

Hanawa, T., Kamahori, H., Maruyama, T., and Shibata, K. 1988, Pub. Astr. Soc. Japan, 40, 171.

Kronberg, P. P., Biermann, P., and Schwab, F. R. 1985, Ap. J., 291, 693.

Lovelace, R. V. E. 1976, Nature, 262, 649.

Lovelace, R. V. E. 1990, in this volume.

Morris, M. 1990, in this volume.

Nakai, N. et al. 1987, Pub. Astr. Soc. Japan, 39, 685.

Parker, E. N. 1966, Ap. J., 145, 811.

Pudritz, R. E. and Norman, C. 1986, Ap. J., 301, 571.

Shibata, K. 1989, in M. Morris (ed.), Proc. IAU Symp. No. 136, The Center of the Galaxy, Reidel, p. 313.

Shibata, K. and Matsumoto, R. 1990, in this volume.

Shibata, K. and Uchida, Y. 1985, Pub. Astr. Soc. Japan, 37, 31.

Shibata, K. and Uchida, Y. 1986, Pub. Astr. Soc. Japan, 38, 631.

Shibata, K. and Uchida, Y. 1987, Pub. Astr. Soc. Japan, 39, 559.

Shibata, K. and Uchida, Y. 1990, Pub. Astr. Soc. Japan, 42, No. 1, in press.

Shibata, K., Tajima, T., and Matsumoto, R. 1990, Ap. J., 350, Feb. 10 issue.

Shibata, K., Tajima, T., Matsumoto, R., Horiuchi, T., Hanawa, T., Rosner, R., and Uchida, Y. 1989, Ap. J., 338, 471. 
Sofue, Y. and Handa, T. 1984, Nature, 310, 567.

Sofue, Y. 1988, in R. E. Pudritz and Fich, M. (eds.), Galactic and Extragalactic Star Formation, Kluwer Academic Pub., p. 409.

Turner, B. E. 1985, Ap. J., 299, 299.

Uchida, Y. and Shibata, K. 1985, Pub. Astr. Soc. Japan,

Uchida, Y. and Shibata, K. 1986, Can. J. Phys., 64, 507.

Uchida, Y., Shibata, K., and Sofue, Y. 1985, Nature, 317, 699.

Uchida, Y., Hamatake, H., Norman, C., and Shibata, K. 1989, in preparation.

Umemura, S., Iki, K., Shibata, K., and Sofue, Y. 1988, Pub. Astr. Soc. Japan, 40, 2.

RUZMAIKIN: You start with a given magnetic field. However, it is possible to generate a magnetic field inside the hydrodynamical jets. What is first: the magnetic field or the hydrodynamical flow?

SHIBATA: Personally, I believe that there was a primordial vertical field in the central regions of galaxies, and this field plays the role of a guiding tube. However, it is possible to create an MHD jet model where the magnetic field is generated inside the jet, if the initial field strength in the corona (halo) is very small.

LESCH: What about the currents in your model?

SHIBATA: The currents produce the toroidal magnetic field. The current loops are closed.

LOVELACE: What is the $\mathrm{t} \rightarrow \infty$ asymptotic configuration of your jets? For long times, mustn't the numerical magnetic diffusivity come in and therefore give reconnection?

SHIBATA: It is not easy to answer this question, because the numerical simulations for long times $(t \rightarrow \infty)$ are very difficult owing to many numerical problems (e.g. large CPU time, numerical error coming from boundary conditions, etc.). In some cases, however, I found that the system tends to be steady and shows (slow) magnetic reconnection on the equatorial plane, although I have not yet studied them in detail. I hope I will be able to answer this question more definitely in the future.

MOUSCHOVIAS: Please forgive the elementary level of my question, but I do not see how a $40 \mu \mathrm{G}$ magnetic field can collimate a $10^{54} \mathrm{erg}$ explosion. Even if I consider a relatively late time, at which the ejecta of the explosion have expanded to a volume $\sim 10 \mathrm{pc}$ across, the energy density in this "fireball" is $\sim 10^{54} \mathrm{ergs} /\left(10 \mathrm{pc} \times 3.10^{18} \mathrm{~cm} / \mathrm{pc}\right)^{3} \sim 1 / 3.10^{-4}$ ergs $/ \mathrm{cm}^{3}$. On the other hand, the magnetic energy density, with the $40 \mu \mathrm{G}$ field you assume, is $\mathrm{B}^{2} / 8 \pi \sim\left(40.10^{-6} \mathrm{G}\right)^{2} / 8 \pi \sim 2 / 3 \cdot 10^{-10} \mathrm{ergs} / \mathrm{cm}^{3}$. You are short by about six orders of magnitude at that radius, and by many more orders of magnitude at earlier phases of the expansion! How do you reconcile this elementary comparison with the results of your calculation, which shows nice collimation of the ejecta by the magnetic field? Are you assuming that the ejecta by themselves leave the point of explosion with 
a high degree of collimation? At the formal level, what is your equation for $\partial \overrightarrow{\mathrm{B}} / \partial t$ ?

SHIBATA: We showed that the outflow ejected from the disk into the halo by the shock wave resulting from a single adiabatic explosion is collimated by the magnetic field with $\equiv 40 \mu \mathrm{G}$ when the outflow radius reaches $\sim 300 \mathrm{pc}$. Thus the energy density in the fireball is $\sim 10^{54} /$ $\left(300 \mathrm{pc} \times 3.10^{18} \mathrm{~cm} / \mathrm{pc}\right)^{3} \sim 10^{-9} \mathrm{erg} / \mathrm{cm}^{3}$. Actually the length of the outflow is already longer than $\sim 300$ pc at this stage because of the density distribution in the disk-halo system, the magnetic field with $\geq 40 \mu \mathrm{G}$ (energy density $\geqq\left(40 \cdot 10^{-6} \mathrm{G}\right)^{2} / 8 \pi \sim 2 / 3 \cdot 10^{-10} \mathrm{erg} / \mathrm{cm}^{3}$ ) is strong enough to collimate the outflow.

VOLK: I certainly agree with Mouschovias' point in general. If in the starburst case you indeed deposit energies of that order into a small region, then you produce simply a thermal explosion; gravity and magnetic field will presumably play little role. I meant this in my talk on galactic winds, where I thought that our Galactic center was playing such a special role - a mini-M82. Collimation, if any, could come through funneling by the high density gas in the Galactic center.

SHIBATA: As I answered to Dr. Mouschovias' question, the radius of the starburst outflow is not $\sim 10$ pc but $\sim 300$ pc (for M82). Then it is possible to collimate the outflow with $E \sim 10^{54} \mathrm{erg}$ by a magnetic field of $\mathrm{B} \approx 40 \mu \mathrm{G}$. Density distribution helps to produce an elongated flow near the disk, but is not sufficient to make a large-scale elongated flow like M82 and Galactic Center Lobe (see Umemura et al. (1988, Publ. Astron. Soc. Japan 40, 25) for comparison of cases with and without magnetic fields). 